PROCEEDINGS OF THE

AMERICAN MATHEMATICAL SOCIETY

Volume 136, Number 7, July 2008, Pages 2351-2357

S 0002-9939(08)09207-1

Article electronically published on March 4, 2008

\title{
DYNAMICS OF THE $w$ FUNCTION AND THE GREEN-TAO THEOREM ON ARITHMETIC PROGRESSIONS IN THE PRIMES
}

\author{
YONG-GAO CHEN AND YING SHI
}

(Communicated by Wen-Ching Winnie Li)

\begin{abstract}
Let $A_{3}$ be the set of all positive integers $p q r$, where $p, q, r$ are primes and possibly two, but not all three of them are equal. For any $n=$ $p q r \in A_{3}$, define a function $w$ by $w(n)=P(p+q) P(p+r) P(q+r)$, where $P(m)$ is the largest prime factor of $m$. It is clear that if $n=p q r \in A_{3}$, then $w(n) \in A_{3}$. For any $n \in A_{3}$, define $w^{0}(n)=n, w^{i}(n)=w\left(w^{i-1}(n)\right)$ for $i=1,2, \ldots$. An element $n \in A_{3}$ is semi-periodic if there exists a nonnegative integer $s$ and a positive integer $t$ such that $w^{s+t}(n)=w^{s}(n)$. We use $\operatorname{ind}(n)$ to denote the least such nonnegative integer $s$. Wushi Goldring [Dynamics of the $w$ function and primes, J. Number Theory 119(2006), 86-98] proved that any element $n \in A_{3}$ is semi-periodic. He showed that there exists $i$ such that $w^{i}(n) \in\{20,98,63,75\}$, ind $(n) \leqslant 4(\pi(P(n))-3)$, and conjectured that ind $(n)$ can be arbitrarily large.

In this paper, it is proved that for any $n \in A_{3}$ we have $\operatorname{ind}(n)=$ $O\left((\log P(n))^{2}\right)$, and the Green-Tao Theorem on arithmetic progressions in the primes is employed to confirm Goldring's above conjecture.
\end{abstract}

\section{INTRODUCTION}

Let

$$
A_{3}=\{n=p q r \mid p, q, r \text { are all primes }\} \backslash\left\{n=p^{3} \mid p \text { is prime }\right\} .
$$

For any $n \in A_{3}$, define a function $w$ by

$$
w(n)=P(p+q) P(p+r) P(q+r),
$$

where $P(m)$ is the largest prime factor of $m$. It is clear that $w\left(A_{3}\right) \subseteq A_{3}$ (see 1. Lemma 2.1]). For any $n \in A_{3}$, define $w^{0}(n)=n, w^{i}(n)=w\left(w^{i-1}(n)\right)$ for $i=1,2, \ldots$, and define the $w$-orbit of $n$ to be the sequence $W(n)=[n, w(n), \ldots$, $\left.w^{i}(n), \ldots\right]$.

Wushi Goldring 1 proved that for any $n \in A_{3}$, there exists $i$ such that $W(n)=$ $\left[n, w(n), \ldots, w^{i-1}(n), \overline{20,98,63,75}\right]$. For $n \in A_{3}$, the periodicity index of $n$ is defined to be the least nonnegative integer $i$ such that $w^{i}(n) \in\{20,98,63,75\}$. Denote the periodicity index of $n$ by ind $(n)$. Wushi Goldring 1$]$ proved that ind $(n) \leqslant$ $4(\pi(P(n))-3)$, and posed several conjectures related to $w(n)$. Two of them are

Received by the editors October 6, 2006, and, in revised form, April 30, 2007.

2000 Mathematics Subject Classification. Primary 11A25, 11A41, 37 B99.

The authors were supported by the National Natural Science Foundation of China, Grants No. 10471064 and 10771103 .

(C)2008 American Mathematical Society Reverts to public domain 28 years from publication 
Conjecture 2.9. $\operatorname{ind}(n)=O(\log \pi(P(n)))$.

Conjecture 2.10. There are sets in $A_{3}$ of arbitrarily large periodicity index.

In this paper we improve the upper bound of ind $(n)$ and employ the Green-Tao Theorem on arithmetic progressions in the primes to confirm Goldring's Conjecture 2.10 .

Theorem 1. For any $n \in A_{3}$, we have ind $(n)=O\left((\log P(n))^{2}\right)$.

Theorem 2. $\operatorname{ind}(n)$ can be arbitrarily large.

Remark. By the Prime Number Theorem, it is clear that $\log (\pi(P(n))) \sim \log P(n)$. By the proofs of Theorems 1 and 2, we believe that Conjecture 2.9 is not true.

\section{Proofs of THEOREMS}

We will make repeated use of the following trivial facts.

Lemma 1. Let $p, q$ be odd primes. Then

(a) $P(p+q) \leq \frac{1}{2}(p+q)$;

(b) if $p+2$ is composite, then $P(p+2) \leq \frac{1}{3}(p+2)$;

(c) if $p+2$ is prime and $p>3$, then $p+4$ must be composite and $P(p+4) \leq$ $\frac{1}{3}(p+4)$.

Lemma 2. Let $X$ be an integer with $X \geqslant 3$ and $\alpha$ be a real number with $0<\alpha<1$. For any $n=p q r \in A_{3}$, where $p, q, r$ are all primes with $p \leqslant X, q \leqslant \alpha X$ and $r \leqslant \alpha X$, there exists $1 \leqslant i \leqslant 3$ such that

$$
P\left(w^{i}(n)\right) \leqslant \frac{1}{4}(\alpha+3) X+4 .
$$

Proof. By the definition of $w$ we have

$$
w(n)=P(p+q) P(p+r) P(q+r) .
$$

If $p \leqslant \alpha X$, then by Lemma 1 ,

$$
P(w(n)) \leqslant \alpha X+2 \leqslant \frac{1}{4}(\alpha+3) X+4 .
$$

Now we may assume that $p>\alpha X$. If $p=3$, then by $q \leqslant \alpha X$ and $r \leqslant \alpha X$ we have $q=r=2$. Then

$$
P(w(n))=5 \leqslant \frac{1}{4}(\alpha+3) X+4 .
$$

Thus we may assume that $p \geqslant 5$ and $p>\alpha X$.

Case 1. $p+2$ is composite.

By Lemma 1 we have

$$
P(p+q) \leqslant\left\{\begin{array}{ll}
\frac{p+2}{3}, & \text { if } q=2, \\
\frac{1+\alpha}{2} X, & \text { if } q \geqslant 3,
\end{array} \quad P(p+r) \leqslant \begin{cases}\frac{p+2}{3}, & \text { if } r=2, \\
\frac{1+\alpha}{2} X, & \text { if } r \geqslant 3,\end{cases}\right.
$$

and

$$
P(q+r) \leqslant \begin{cases}\alpha X, & \text { if } q \geqslant 3 \text { and } r \geqslant 3, \\ \alpha X+2, & \text { if } q=2 \text { or } r=2 .\end{cases}
$$

Hence

$$
P(w(n)) \leqslant \frac{1}{4}(\alpha+3) X+4 .
$$


Case 2. $p+2$ is prime. Since $p>3$ and $p, p+2$ are both primes, we have $3 \mid p+4$.

Subcase 2.1. $q, r$ are both odd primes. By Lemma 1 we have

$$
P(w(n)) \leqslant \max \left\{\frac{1+\alpha}{2} X, \alpha X\right\} \leqslant \frac{1}{4}(\alpha+3) X+4 .
$$

Subcase 2.2. $q>r=2$. Then $w(n)=(p+2) P(p+q) P(q+2)$. Let $p_{1}=p+2, q_{1}=$ $P(p+q), r_{1}=P(q+2)$. Since $p_{1} \leqslant p+2, q_{1} \leqslant \frac{1}{2}(1+\alpha) X, r_{1} \leqslant \alpha X+2$, and $r_{1}$ is odd, by Lemma 1 we have

$$
\begin{aligned}
& P\left(p_{1}+q_{1}\right) \leqslant \begin{cases}\frac{p+4}{3}, & \text { if } q_{1}=2, \\
\frac{3+\alpha}{4} X+1, & \text { if } q_{1} \text { is odd, }\end{cases} \\
& P\left(q_{1}+r_{1}\right) \leqslant \begin{cases}\alpha X+4, & \text { if } q_{1}=2, \\
\frac{1+3 \alpha}{4} X+1, & \text { if } q_{1} \text { is odd },\end{cases}
\end{aligned}
$$

and

$$
P\left(p_{1}+r_{1}\right) \leqslant \frac{1+\alpha}{2} X+2 .
$$

Hence

$$
P\left(w^{2}(n)\right) \leqslant \frac{1}{4}(\alpha+3) X+4
$$

Similarly, when $r>q=2$, we can get the same conclusion.

Subcase 2.3. $q=r=2$. Then

$$
w^{3}(n)=P(p+4) \cdot P^{2}(P(p+4)+p+2) .
$$

By Lemma 1 we have

$$
P(p+4) \leqslant \frac{p+4}{3}, P(P(p+4)+p+2) \leqslant \frac{p+2+(p+4) / 3}{2}=\frac{2 p+5}{3} .
$$

Hence

$$
P\left(w^{3}(n)\right) \leqslant \frac{1}{4}(\alpha+3) X+4 .
$$

This completes the proof of Lemma 2 .

Corollary 3. Let $X$ be an integer with $X \geqslant 3$ and $\alpha$ be a real number with $0<$ $\alpha<1$. For $n=p q r \in A_{3}$ with $p \geqslant q \geqslant r \geqslant 3, p \leqslant X$ and $r \leqslant \alpha X$, there exists $2 \leqslant i \leqslant 4$ such that

$$
P\left(w^{i}(n)\right) \leqslant \frac{1}{8}(\alpha+7) X+4 .
$$

Proof. Let

$$
p_{1}=P(p+q), q_{1}=P(p+r), r_{1}=P(q+r) .
$$

By Lemma 1 we have

$$
p_{1} \leqslant X, q_{1} \leqslant \frac{1+\alpha}{2} X, r_{1} \leqslant \frac{1+\alpha}{2} X .
$$

Since $w(n)=P(p+q) P(p+r) P(q+r)$, by Lemma 2 , there exists $1 \leqslant j \leqslant 3$ such that

$$
P\left(w^{j}(w(n))\right) \leqslant \frac{3+(1+\alpha) / 2}{4} X+4 .
$$

Therefore, there exists $2 \leqslant i \leqslant 4$ such that

$$
P\left(w^{i}(n)\right) \leqslant \frac{1}{8}(\alpha+7) X+4 .
$$


This completes the proof of Corollary 3.

Lemma 4. For any $n \in A_{3}$ there exists $1 \leqslant i \leqslant 2 \log (P(n)+2)+6$ such that

$$
P\left(w^{i}(n)\right) \leqslant \frac{15}{16} P(n)+6 .
$$

Proof. For any $n=p q r \in A_{3}$ with $p \geqslant q \geqslant r \geqslant 2$, we have

$$
w(n)=P(p+q) P(p+r) P(q+r) .
$$

Case 1. $p, q, r$ are all odd primes.

Subcase 1.1. At most one of $\frac{1}{2}(p+q), \frac{1}{2}(p+r)$, and $\frac{1}{2}(q+r)$ is an odd prime.

If there is an odd prime, then this odd prime is not larger than $p$, but the other two prime factors of $w(n)$ are not larger than $\frac{1}{2} p$. Otherwise all of $P(p+q), P(p+r)$ and $P(q+r)$ are not larger than $\frac{1}{2} p$. Hence, by Lemma 2 , there exists $1 \leqslant i \leqslant 4$ such that

$$
P\left(w^{i}(n)\right) \leqslant \frac{1}{4}\left(3+\frac{1}{2}\right) p+4=\frac{7}{8} p+4 .
$$

Subcase 1.2. Precisely two of $\frac{1}{2}(p+q), \frac{1}{2}(p+r)$, and $\frac{1}{2}(q+r)$ are odd primes.

Firstly, we suppose that $\frac{1}{2}(p+q)$ and $\frac{1}{2}(p+r)$ are odd primes. Let

$$
p_{1}=P(p+q)=\frac{p+q}{2}, \quad q_{1}=P(p+r)=\frac{p+r}{2}, \quad r_{1}=P(q+r) .
$$

Since $p_{1}+q_{1}=p+\frac{q+r}{2}$ is even, $1 \neq \frac{q+r}{2}$ is odd. Thus $3 \leqslant r_{1}=P(q+r) \leqslant \frac{1}{2} p$. By Corollary 3 , there exists $1 \leqslant i \leqslant 5$ such that

$$
P\left(w^{i}(n)\right) \leqslant \frac{1}{8}\left(7+\frac{1}{2}\right) p+4=\frac{15}{16} p+4 .
$$

Similarly, we have the same conclusion for the other two cases: (a) $\frac{1}{2}(p+q)$ and $\frac{1}{2}(q+r)$ are odd primes; (b) $\frac{1}{2}(p+r)$ and $\frac{1}{2}(q+r)$ are odd primes.

Subcase 1.3. All of $\frac{1}{2}(p+q), \frac{1}{2}(p+r)$, and $\frac{1}{2}(q+r)$ are odd primes.

Let

$$
\begin{aligned}
& p_{1}=\frac{p+q}{2}, q_{1}=\frac{p+r}{2}, r_{1}=\frac{q+r}{2}, \\
& p_{i}=\frac{p_{i-1}+q_{i-1}}{2}, q_{i}=\frac{p_{i-1}+r_{i-1}}{2}, r_{i}=\frac{q_{i-1}+r_{i-1}}{2}(i=2,3, \ldots) .
\end{aligned}
$$

Assume that for $i=1,2, \ldots, k$, all of $p_{i}, q_{i}, r_{i}$ are odd primes, and at least one of $p_{k+1}, q_{k+1}, r_{k+1}$ is not an odd prime. Since

$$
1 \leqslant p_{k}-r_{k}=\frac{p_{k-1}-r_{k-1}}{2}=\cdots=\frac{p-r}{2^{k}},
$$

we have $k \leqslant 2 \log p$. By Subcases 1.1 and 1.2 , there exists $1 \leqslant j \leqslant 5$ such that

$$
P\left(w^{j}\left(p_{k} q_{k} r_{k}\right)\right) \leqslant \frac{15}{16} p_{k}+4 \leqslant \frac{15}{16} p+4
$$

Thus

$$
P\left(w^{k+j}(n)\right) \leqslant \frac{15}{16} p+4 .
$$

Hence, if $p, q, r$ are all odd primes, there exists $1 \leqslant i \leqslant 2 \log p+5$ such that

$$
P\left(w^{i}(n)\right) \leqslant \frac{15}{16} p+4 .
$$


Case 2. $p \geqslant q>r=2$.

Now we note that $P(p+2)$ and $P(q+2)$ are odd primes.

Subcase 2.1. $P(p+q)$ is an odd prime.

As in Case 1 , there exists $1 \leqslant i \leqslant 2 \log (p+2)+6$ such that

$$
P\left(w^{i}(n)\right) \leqslant \frac{15}{16}(p+2)+4 \leqslant \frac{15}{16} p+6 .
$$

Subcase 2.2. $P(p+q)=2$.

- If at least one of $p+2$ and $q+2$ is not prime, then by Lemma 1 we have

$$
P(p+2) \leqslant \frac{p+2}{3} \quad \text { or } \quad P(q+2) \leqslant \frac{p+2}{3} .
$$

Noting that $2 \leqslant \frac{1}{2}(p+2)$, by Lemma 2 there exists $1 \leqslant i \leqslant 4$ such that

$$
P\left(w^{i}(n)\right) \leqslant \frac{1}{4}\left(3+\frac{1}{2}\right)(p+2)+4=\frac{7}{8}(p+2)+4 .
$$

- If $p+2, q+2$ are both primes, then $p+4$ is odd composite and $q+4$ is odd composite or 7 , and

$$
w^{2}(n)=P(p+4) P(q+4) P(p+q+4) .
$$

Since $P(p+q)=2$, we have $4 \mid p+q+4$. So

$$
P(p+q+4) \leqslant \frac{p+q+4}{4} .
$$

By Lemma 1 we have

$$
P(p+4) \leqslant \frac{p+4}{3}, P(q+4) \leqslant \max \left\{7, \frac{p+4}{3}\right\} .
$$

Hence

$$
P\left(w^{2}(n)\right) \leqslant \frac{15}{16} p+6 .
$$

Case 3. $q=r=2$. Then $n=2^{2} p$. By Lemma 2 with $\alpha=\frac{2}{3}$ and $X=p$, there exists $1 \leqslant i \leqslant 3$ such that

$$
P\left(w^{i}(n)\right) \leqslant \frac{11}{12} p+4 .
$$

This completes the proof of Lemma 4.

Proof of Theorem 1. For any $n=p q r \in A_{3}$ let $i_{0}=0$ and $P(n)=p$. By Lemma 4 there exist positive integers $i_{1}<i_{2}<\cdots$ such that

$$
P\left(w^{i_{k}}(n)\right) \leqslant \frac{15}{16} P\left(w^{i_{k-1}}(n)\right)+6, \quad k=1,2, \ldots
$$

and

$$
i_{k}-i_{k-1} \leqslant 2 \log \left(P\left(w^{i_{k-1}}(n)\right)+2\right)+6, \quad k=1,2, \ldots .
$$

By (1) we have

$$
\begin{aligned}
P\left(w^{i_{k}}(n)\right) & \leqslant \frac{15}{16} P\left(w^{i_{k-1}}(n)\right)+6 \leqslant\left(\frac{15}{16}\right)^{2} P\left(w^{i_{k-2}}(n)\right)+6\left(\frac{15}{16}\right)+6 \leqslant \cdots \\
& \leqslant\left(\frac{15}{16}\right)^{k} P\left(w^{i_{0}}(n)\right)+6\left(\frac{15}{16}\right)^{k-1}+6\left(\frac{15}{16}\right)^{k-2}+\cdots+6\left(\frac{15}{16}\right)+6 \\
& <\left(\frac{15}{16}\right)^{k} p+96 .
\end{aligned}
$$


If

then

$$
k \geqslant \frac{\log p}{\log 16-\log 15}
$$

$$
P\left(w^{i_{k}}(n)\right) \leqslant\left(\frac{15}{16}\right)^{k} p+96 \leqslant 97 .
$$

For each $m \in A_{3}$ with $P(m) \leqslant 97$, by [1, Theorem 1.1] there exists $j_{m}$ such that

$$
w^{j_{m}}(n) \in\{20,98,63,75\} .
$$

Let $c=\max \left\{j_{m} \mid m \in A_{3}, P(m) \leq 97\right\}$. Then there exists $j$ with $1 \leqslant j \leqslant c$ such that

$$
w^{i_{k}+j}(n)=w^{j}\left(w^{i_{k}}(n)\right) \in\{20,98,63,75\} .
$$

By [1, Corollary 2.6] we have

$$
P\left(w^{i_{k}}(n)\right) \leqslant p+4, \quad k=1,2, \ldots
$$

By (2) and (3) we have

$$
i_{k}-i_{k-1} \leqslant 2 \log (p+6)+6, \quad k=1,2, \ldots
$$

Thus

$$
i_{k} \leqslant 2 k \log (p+6)+6 k, \quad k=1,2, \ldots
$$

Take an integer $k$ with

$$
k \geqslant \frac{\log p}{\log 16-\log 15}>k-1
$$

Then

$$
\operatorname{ind}(n) \leqslant i_{k}+j \leqslant 2 k \log (p+6)+6 k+c \ll(\log p)^{2} .
$$

That is,

$$
\operatorname{ind}(n)=O\left((\log p)^{2}\right) .
$$

This completes the proof of Theorem 1.

Finally, we employ the Green-Tao Theorem on arithmetic progressions in the primes to confirm Goldring's Conjecture 2.10 [1]; i.e., ind $(n)$ can be arbitrarily large.

Proof of Theorem 2. By the Green-Tao Theorem on arithmetic progressions in the primes (see [2]), for any positive integer $k \geqslant 7$, there exist two positive integers $a, d$ such that $a+i d\left(0 \leqslant i \leqslant 2^{k}\right)$ are all primes. Let

$$
p_{0}=a+2^{k} d, q_{0}=a+2^{k-1} d, r_{0}=a,
$$

and

$$
p_{i}=\frac{p_{i-1}+q_{i-1}}{2}, q_{i}=\frac{p_{i-1}+r_{i-1}}{2}, r_{i}=\frac{q_{i-1}+r_{i-1}}{2}, i=1,2, \ldots, k-1 .
$$

Then $p_{i}, q_{i}$ and $r_{i}(i=0,1,2, \ldots, k-1)$ are in the arithmetic progression $a+i d(0 \leqslant$ $\left.i \leqslant 2^{k}\right)$. Hence $p_{i}, q_{i}$ and $r_{i}(i=0,1,2, \ldots, k-1)$ are all primes. Let $n=p_{0} q_{0} r_{0}$. Then $w^{i}(n)=p_{i} q_{i} r_{i}(i=1,2, \ldots, k-1)$. Noting that

$$
p_{i}+q_{i}+r_{i}=p_{0}+q_{0}+r_{0}>2^{k}
$$

we have $w^{i}(n) \notin\{20,63,75,98\}(i=1,2, \ldots, k-1)$. So ind $(n) \geqslant k$. This completes the proof of Theorem 2 . 


\section{ACKNOWLEDGEMENT}

We would like to thank the referee for his/her kind comments.

\section{REFERENCES}

[1] Wushi Goldring, Dynamics of the $w$ function and primes, J. Number Theory 119 (2006), 86-98. MR.2228951 (2007a:11010)

[2] Ben Green and Terence Tao, The primes contain arbitrarily long arithmetic progressions, to appear in Ann. Math.

Department of Mathematics, Nanjing Normal University, Nanjing 210097, People's Republic of China

E-mail address: ygchen@njnu.edu.cn

Department of Mathematics, Nanjing Normal University, Nanjing 210097, People's Republic of China 